\title{
Nei luoghi della Guerra e della Resistenza a Parma. Un itinerario di studio condotto da studenti per studenti.
}

Alunni delle scuole superiori, dopo essere stati appositamente formati, fanno da guida a classi terze delle scuole medie, accompagnandole in due percorsi cittadini di circa 10 "tappe" ciascuno nei luoghi della memoria. Durante questi itinerari le guide introducono temi e avvenimenti di rilievo relativi alla Seconda guerra mondiale e alla Resistenza. I partecipanti realizzano brevi testi che sono raccolti in un quaderno distribuito durante un incontro organizzato entro la fine dell'anno scolastico.

Trained students (16-19 years old) serve as guides to other students (12-13 years old), accompanying them in city walks composed of about 10 "stops" in the places of memory. During these tours the guides introduce themes and major events related to World War 2 and Resistance. After that the participants realize short texts which are collected in a book distributed during a meeting organized by the end of the school year.

"Nei luoghi della guerra e della Resistenza" è un progetto che prevede percorsi guidati durante i quali ragazzi delle scuole superiori accompagnano classi delle scuole medie nei luoghi della memoria della propria città. L'iniziativa è rivolta a studenti di età compresa tra i 12 e i 19 anni. Il programma è iniziato nel 2006 su idea e impulso dell'Istituto storico della Resistenza e dell'età contemporanea di Parma (Isrec) e del Servizio diritto allo studio (Assessorato alla Pubblica istruzione) del Comune di Parma; nel 2014 è giunto alla nona edizione consecutiva. Annualmente 20-23 alunni volontari delle classi terze, quarte e quinte di scuole secondarie di secondo grado partecipano a laboratori pomeridiani, organizzati dall'Isrec fuori dall'orario scolastico, riguardanti temi e fatti principali relativi alla Resistenza e alla Seconda guerra mondiale. Durante le prime due lezioni teoriche i ragazzi vengono istruiti riguardo l'armistizio, la nascita della Resi- 
stenza armata e non, l'invasione tedesca, i bombardamenti alleati, la repressione nazifascista, la deportazione, la Liberazione, la memoria nel dopoguerra. Viene consegnato loro il materiale necessario per approfondire i soggetti trattati e poterli esporre agli altri studenti: saggi, documenti, immagini. Segue un momento di formazione pratica delle future guide, che, durante altri due incontri, vengono accompagnate nei percorsi previsti dal progetto.

Nel tempo sono stati sviluppati due differenti itinerari, diversificati ma complementari, della durata di della durata di 2 ore ciascuno. Per diversi anni l'iniziativa si è svolta su un unico percorso, nel centro storico della città. Nel 2010 si è aggiunto un secondo itinerario, simile nei temi ma con luoghi differenti, nel quartiere storico popolare Oltretorrente. Ogni percorso è composto da circa 10 tappe; sono stati selezionati luoghi ritenuti più significativi per meglio comprendere ciò che accadde durante la guerra in città. Tra i punti di sosta vi sono rifugi antiaerei, edifici che furono bombardati, monumenti, lapidi commemorative, sedi di caserme, siti in cui vi furono arresti ed eccidi, zone in cui avvennero battaglie nei giorni successivi l'armistizio, sedi delle istituzioni fasciste e naziste, abitazioni di partigiani e di deportati. A monumenti frequentati e conosciuti sono affiancati luoghi dimenticati, non segnalati, tracce ormai invisibili del passato.

L'iter di formazione si conclude con due incontri di verifica finali durante i quali i ragazzi che hanno frequentato le lezioni possono mettersi alla prova e verificare la propria preparazione. Il progetto viene proposto a classi terze delle scuole secondarie di primo grado, in questo modo gli insegnanti possono affrontare, o hanno già affrontato, gli argomenti relativi agli itinerari durante le loro lezioni.

Le visite guidate avvengono la mattina in orario scolastico. Dopo aver esposto brevemente il contesto storico, due guide affiancate da un tutor accompagnano una classe in uno dei due percorsi previsti. All'inizio di ogni sosta i due accompagnatori introducono la peculiarità della tappa e coinvolgono i ragazzi facendo loro leggere le lapidi commemorative, mostrando immagini, ponendo domande. In questo modo i ragazzi possono riscoprire, reinterpretare, decifrare attivamente le strade e i luoghi della propria città. Durante gli anni scolastici dal 2005/2006 al $2013 / 2014$ sono state effettuate tra le 18 e le 23 visite guidate annuali tra la fine di marzo all'inizio di maggio. Nel 2010 e nel 2011 furono filmate sia la formazione delle guide che i percorsi guidati. Con il materiale girato furono prodotti filmati che erano distribuiti nelle scuole e mandati in onda da televisioni locali, consentendo di ampliare i fruitori del progetto.

Terminato il programma delle visite viene chiesto agli studenti che hanno fatto da guida di scrivere brevi testi sulle diverse soste dei percorsi. Contemporaneamente gli studenti delle scuole medie rielaborano attraverso lettere, poesie, disegni, dia- 
ri, riflessioni la propria esperienza. Dai materiali raccolti, integrati con fotografie e documenti utilizzati durante i laboratori e visite, viene ricavato un quaderno didattico. Il quaderno viene distribuito insieme ai filmati entro la fine di maggio nelle scuole, durante un incontro appositamente organizzato a cui partecipano sia rappresentanze degli studenti delle classi coinvolte che dei ragazzi che hanno svolto il ruolo di guide. Durante l'appuntamento intervengono uno dei tutor dell'Isrec che ha seguito il progetto, alcuni partecipanti delle scuole superiori e diversi studenti delle scuole medie, che leggono i propri elaborati. Al termine della presentazione il quaderno viene distribuito a tutti i partecipanti.

"Nei luoghi della guerra e della Resistenza" è un'iniziativa finanziata dal Comune di Parma, dall'Isrec e da sponsor privati: non ha, quindi, alcun costo per i partecipanti. In ogni edizione si presentano difficoltà organizzative, legate principalmente alla necessità di trovare date utili per programmare guide e laboratori, visti i diversi soggetti impegnati. Difficoltà che si risolvono grazie al pragmatismo, alla disponibilità e ai buoni rapporti con scuole e insegnanti.

Il progetto è nato da una riflessione riguardo la frequentazione da parte dei giovani delle vie del centro città, costellate di luoghi e monumenti densi di memoria e, al contempo, la scarsa appetibilità dello studio della storia. Per avvicinare e coinvolgere i ragazzi in un racconto storico sono stati uniti l'ascolto di una narrazione breve ma esaustiva di un periodo storico con la piacevolezza di un itinerario pedonale nelle vie della città.

Un ulteriore criterio deciso per avvicinare gli studenti e attirare la loro attenzione è stato quello dell'età degli accompagnatori. Per fare da guida a ragazzi di $12 \mathrm{e}$ 13 anni è stata chiesta la disponibilità di studenti di età poco superiore, compresa tra i 16 e i 19 anni. Gli alunni tendono ad ascoltare con maggiore interesse e partecipazione le narrazioni dei compagni di studi, con i quali possono instaurare un rapporto paritario. D'altro canto gli studenti delle scuole superiori hanno accettato di buon grado il loro ruolo di accompagnatori, accogliendo l'invito a partecipare a un periodo di formazione presso l'Isrec relativamente lungo e impegnativo. Il successo di "Nei luoghi della Guerra e della Resistenza" è testimoniato dalle numerose richieste di partecipazione che giungono ogni anno, sia da parte dei ragazzi che vogliono diventare guide, sia dagli insegnati delle scuole medie. Il valore e la peculiarità di questo progetto, oltre alla sua funzione di studio e di conoscenza della storia contemporanea, consiste, dunque, nelle sue modalità di attuazione: sono coinvolti, in un'attività corale, alunni di età differenti ed esperti nella storia del territorio al fine di favorire una nuova forma di ricerca e studio. 